\title{
Patientenerwartungen optimieren: Beschreibung einer präoperativen Kurzintervention am Beispiel von Patienten vor einer Bypass-Operation
}

\author{
Stefan Salzmann $^{a}$ Johannes Laferton $^{\mathrm{b}, \mathrm{c}}$ Charlotte Auer ${ }^{\mathrm{a}} \quad$ Meike Shedden-Mora $^{\mathrm{d}}$ \\ Katrin Wambach ${ }^{a}$ Winfried Rief ${ }^{a}$
}

${ }^{a}$ Arbeitsgruppe Klinische Psychologie und Psychotherapie, Fachbereich Psychologie, Philipps-Universität Marburg, Marburg, Deutschland;

${ }^{b}$ Psychologische Hochschule Berlin, Berlin, Deutschland;

${ }^{c}$ Lehrstuhl für Klinische Psychologie und Psychotherapie, Institut für Psychologie, Friedrich-Alexander-Universität Erlangen-Nürnberg, Erlangen, Deutschland;

${ }^{\mathrm{d}}$ Institut und Poliklinik für Psychosomatik und Psychotherapie, Universitätsklinikum Hamburg-Eppendorf, Hamburg, Deutschland

\section{Schlüsselwörter \\ Erwartungen - Placeboeffekt · Koronare Bypass-Operation . \\ Psychologische Intervention · Klinische Studie}

\section{Zusammenfassung}

Hintergrund: Patientenerwartungen gelten als wichtige Wirkmechanismen bei psychotherapeutischen und medizinischen Behandlungen und spielen für die Genesung eine große Rolle. Vor diesem Hintergrund wurde eine psychologische präoperative Kurzintervention zur Erwartungsoptimierung entwickelt, um den Genesungsprozess nach Herzoperationen positiv zu beeinflussen. Dieser Artikel beschreibt die psychologische Kurzintervention (EXPECT) und stellt Evaluationsergebnisse der Intervention aus Patientensicht dar. Patienten und Methoden: 124 herzchirurgische Patienten wurden randomisiert und einer von drei Interventionen zugeteilt: a) Erwartungsoptimierung, b) supportive Therapie oder c) ausschließlich medizinische Standardbehandlung. In der Erwartungsintervention, die 2 Sitzungen und 2 Telefonate vor der Operation umfasst, wurden systematisch verhaltens- und behandlungsbezogene Ergebniser wartungen optimiert. Die Patienten wurden mithilfe von Fragebögen zu ihrer Zufriedenheit mit der Intervention befragt. Mittelwerte wurden berechnet. Ergebnisse: Die Zufriedenheit der Patienten mit beiden psychologischen Interventionen war im Mittel hoch bis sehr hoch. In der Erwartungsinterventionsgruppe fühlten sich die Patienten noch besser informiert und erwarteten einen positiveren Heilungsverlauf als die Patienten, die eine supportive Therapie erhielten. Ein Großteil der Patienten erlebte die Interventionen als hilfreich und erwartete durch diese positivere Ergebnisse, was sich auch im längsschnittlichen Verlauf bestätigte. Somit können präoperative Interventionen bei herzchirurgischen Eingriffen das bestehende Behandlungsangebot sinnvoll erweitern. Schlussfolgerungen: Die Optimierung von verhaltens- und ergebnisbezogenen Patientenerwartungen vor der eigentlichen Intervention kann zu einer Verbesserung von Behandlungsergebnissen beitragen. Eine solche psychologische Intervention wird gut angenommen und ist im Rahmen eines herzchirurgischen Settings durchführbar. Die Förderung positiver Erwartungen und die Veränderung ungünstiger Ausgangserwartungen von Patienten sollten sowohl bei psychotherapeutischen als auch bei herzchirurgischen Behandlungen stärker berücksichtigt werden.

(c) 2018 S. Karger GmbH, Freiburg

Zur besseren Lesbarkeit wird in dieser Arbeit ausschließlich die männliche Form verwendet. Gemeint sind jedoch stets Personen männlichen und weiblichen Geschlechts.

\section{Keywords}

Expectations - Placebo effect - Coronary bypass surgery .

Psychological intervention · Clinical trial

\section{Summary}

Optimizing Patients' Expectations: Description of a Brief Preoperative Intervention for Patients Undergoing Coronary Artery Bypass Graft Surgery

Background: Patients' expectations are considered to be important mechanisms in psychotherapeutic and medical treatments and play a crucial role in recovery. Therefore, a short preoperative psychological intervention targeting patients' expectations was developed to facilitate patients' recovery after heart surgery. This article describes the short psychological intervention (EXPECT) and reports patients' evaluation of the intervention. Patients and Methods: 124 heart surgery patients were randomized and assigned to 1 of 3 interventions: a) optimizing expectations, b) supportive therapy, or c) standard medical care only. The expectation intervention incorporated 2 sessions in person and 2 telephone calls to systematically optimize behavior- and treatment-related outcome expectations. Subsequently, patients evaluated the intervention using questionnaires. Mean scores were calculated. Results: Patients' satisfaction regarding both interventions was rated high to very high on average. Patients undergoing the expectation intervention felt better informed and expected a more positive recovery process compared to the supportive-therapy group. Most patients rated the interventions as being helpful and expected more positive outcomes due to the interventions, which was supported by the longitudinal study results. Preoperative psychological interventions might be a reasonable add-on in the treatment of heart surgery patients. Conclusions: Optimizing patients' behavior- and treatment-related outcome expectations prior to surgery can contribute to more positive treatment outcomes. Such psychological interventions are well accepted by patients and feasible for use in heart surgery settings. Fostering positive expectations and altering negative baseline expectations should be considered in both psychotherapy and treatment of heart surgery patients.

\section{KARGER}

(c) 2018 S. Karger GmbH, Freiburg 


\section{Theoretischer Hintergrund}

Die Relevanz von Patientenerwartungen in der Behandlung sowohl psychischer als auch körperlicher Erkrankungen wird durch Erkenntnisse aus der Placeboforschung immer deutlicher [Rief und Glombiewski, 2016; Rief et al., 2017]. Ansätze zur systematischen Nutzung dieses Wissens sind jedoch noch rar [Enck et al., 2013]. Medizinische Behandlungseffekte setzen sich aus spezifischen und unspezifischen Faktoren zusammen. Ein spezifischer Faktor kann beispielsweise der pharmakologische Wirkstoff eines Medikaments sein, wobei unspezifische Faktoren (z.B. Patientenerwartungen im Hinblick auf das Behandlungsergebnis) ausschlaggebend für Placeboeffekte sind. Erwartungen werden neben der ArztPatienten-Interaktion und Konditionierungsprozessen als einer der wichtigsten Wirkmechanismen von Placeboeffekten konzeptualisiert [Schedlowski et al., 2015]. Placeboeffekte beeinflussen dabei nicht nur subjektive Maße (z.B. Lebensqualität), sondern auch objektive Maße wie beispielsweise Immunparameter [Schedlowski et al., 2015]. So beruht ein Großteil der Wirksamkeit von Antidepressiva auf dem Placeboeffekt [Kirsch, 2016; Shedden-Mora et al., 2011].

Patientenerwartungen sagen auch bei psychotherapeutischen Interventionen den Behandlungserfolg vorher [Constantino et al., 2011] und gelten als einer der wichtigsten allgemeinen Wirkfaktoren von Psychotherapie [Wampold, 2015; Wampold et al., 2005]. Beispielsweise wird ein Patient voraussichtlich nur dann von einer psychotherapeutischen Behandlung profitieren, wenn er auf der Basis einer glaubhaften Erklärung für seine Probleme (z.B. ein nachvollziehbares Krankheitsmodell) generell eine Veränderung seiner gegenwärtigen Situation erwartet. Spezifischer betrachtet ist eine erfolgreiche Psychotherapie nur dann wahrscheinlich, wenn ein Patient erwartet, dass ihm sein Therapeut helfen wird und die durchzuführenden Interventionen hilfreich sein werden, und wenn der Patient langfristig davon ausgeht, seine Probleme selbst positiv beeinflussen zu können [Wampold, 2015].

Selbst bei sehr invasiven medizinischen Prozeduren wie einer aortokoronaren Bypass-Operation hängen die Behandlungsergebnisse nicht allein von den chirurgischen Fähigkeiten oder medizinischen Faktoren ab [Hawkes et al., 2006; Jonas et al., 2015]. Studien zeigen, dass auch hier psychologische Faktoren eine wichtige Rolle für die Genesung und die postoperative Lebensqualität von Herzpatienten spielen [Hawkes et al., 2006; Auer et al., 2016]. Bereits präoperativ sind positive Erwartungen unabhängig von medizinischen Risikofaktoren (z.B. Pumpfunktion des Herzens) wichtige Prädiktoren für eine höhere Lebensqualität, eine geringere Depressivität und eine geringere krankheitsbedingte Beeinträchtigung nach einer Bypass-Operation [Juergens et al., 2010]. Dieser Zusammenhang zwischen präoperativen Erwartungen und postoperativer Lebensqualität - unabhängig von der Art der Operation und Krankheitsschwere - wurde auch in einer Meta-Analyse festgestellt [Auer et al., 2016]. Optimistischere Einstellungen, also generalisierte positive Ergebniserwartungen, gehen mit geringeren Rehospitalisierungsraten nach einer Bypass-Operation einher [Scheier et al., 1999] und sagen sogar Langzeit-Überlebensraten bei Herzpati- enten voraus [Barefoot et al., 2011]. Da Herzerkrankungen weltweit eine der häufigsten Ursachen für eine eingeschränkte Lebensqualität, Erwerbsunfähigkeit und Todesfälle sind sowie massive Kosten für das Gesundheitssystem verursachen [Murray und Lopez, 2013; Go et al., 2014], erscheint eine Erwartungsoptimierung (optimizing expectations) mit dem Ziel, die Behandlungsergebnisse nach einer Bypass-Operation - unabhängig von den medizinischen Faktoren - zu verbessern, sehr relevant.

Mit bisherigen präoperativen psychologischen Interventionen konnten Risikofaktoren verändert, die körperliche Fitness sowie das Wissen über die eigene Erkrankung verbessert [Furze et al., 2009] und Erwartungen verändert werden [Broadbent et al., 2009a]. Allerdings ist die generelle Wirksamkeit von präoperativen Interventionen bei herzchirurgischen Patienten noch nicht eindeutig geklärt [Guo, 2015], und es gibt bisher kaum Studien, in denen versucht wurde, spezifisch präoperative Erwartungen herzchirurgischer Patienten zu optimieren.

Im Rahmen der PSY-HEART-Studie (Psychologische Intervention für Herzpatienten) wurde deshalb eine kurze präoperative psychologische Intervention mit dem primären Ziel entwickelt, durch die Optimierung von Patientenerwartungen den Heilungsverlauf zu verbessern [Laferton et al., 2013]. Die Ergebnisse der Studie wurden kürzlich veröffentlicht und zeigen, dass Patienten von dieser zusätzlichen präoperativen psychologischen Intervention profitierten, indem sie 6 Monate nach der Operation weniger beeinträchtigt waren (verglichen mit Patienten, die nur eine medizinische Standardbehandlung erhielten), eine höhere Lebensqualität berichteten und sogar geringere Entzündungsparameter aufwiesen als Patienten in der Standardbehandlungsbedingung [Rief et al., 2017]. Darüber hinaus konnten die physiologische Stressreaktion nach der Operation verringert [Salzmann et al., 2017] und die Dauer des Krankenhausaufenthalts verkürzt werden [Auer et al., 2017]. Die erwartungsoptimierende Intervention zeigte sich auch gegenüber einer aktiven supportiven Kontrollgruppe hinsichtlich der mentalen Lebensqualität und der Arbeitsfähigkeit überlegen [Rief et al., 2017]. Dabei wurde die Erwartungsmanipulation als erfolgreich eingeschätzt, da beispielsweise die persönliche Kontrollerwartung, also die Erwartung, selbst Einfluss auf die eigene Herzkrankheit zu nehmen, nach der erwartungsoptimierenden Intervention im Vergleich zur Ausgangsmessung signifikant höher war [Rief et al., 2017].

In diesem Artikel sollen die erwartungsoptimierende Intervention und deren Entwicklung genauer beschrieben sowie die Zufriedenheit der Patienten mit den präoperativen psychologischen Interventionen dargestellt und diskutiert werden.

\section{Methode}

Studiendesign

Um zu überprüfen, ob eine präoperative psychologische Kurzintervention zur Erwartungsoptimierung den Genesungsprozess nach einer aortokoronaren Bypass-Operationen positiv beeinflussen kann, wurden Patienten randomisiert und 1 von 3 Bedingungen zugeordnet: entweder einer ausschließlich medizinischen Standardbehandlung (standard medical care; SMC) oder 1 von 2 zusätzli- 


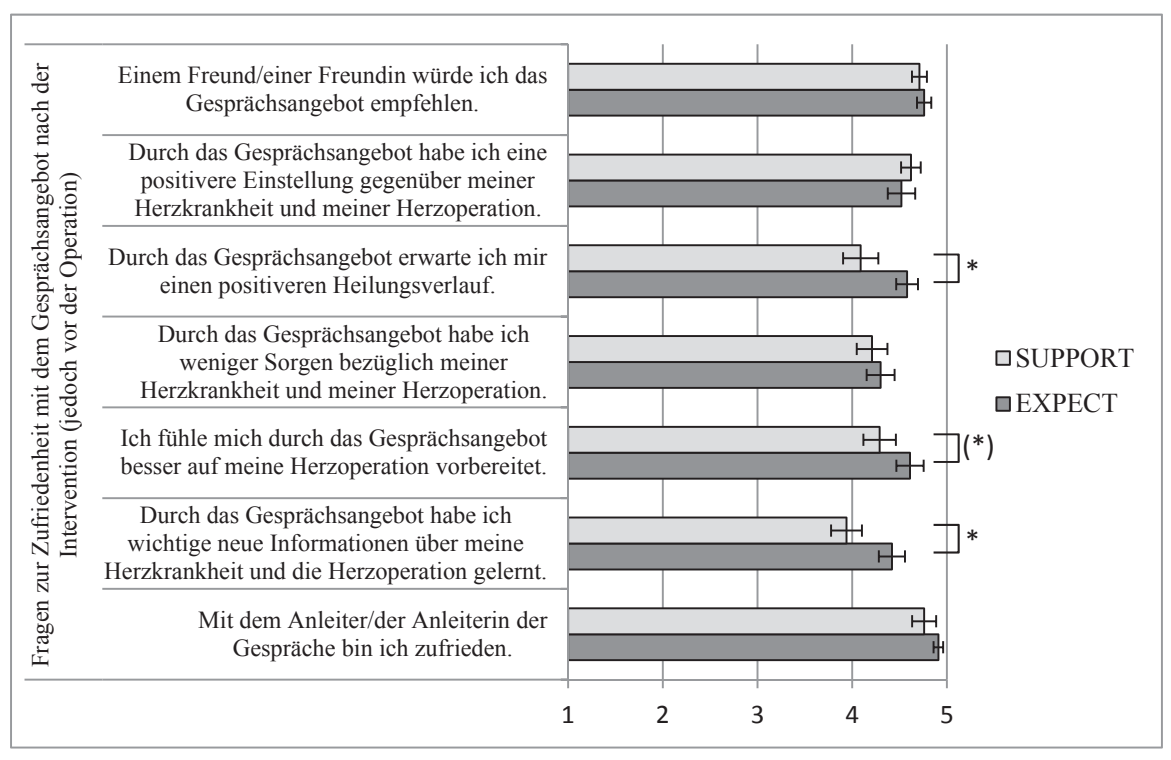

Abb. 1. Einschätzung der Zufriedenheit mit dem Gesprächsangebot / der Intervention nach der psychologischen Intervention, jedoch vor der Operation (oben), und 6 Monate nach der Operation (unten), dargestellt mit Mittelwerten (Standardfehler des Mittelwerts) zum Vergleich zwischen beiden Gruppen (Skala 1-5; $1=$ stimme absolut nicht $\mathrm{zu}, 2$ = stimme weniger $\mathrm{zu}, 3$ = weder noch, $4=$ stimme etwas $\mathrm{zu}, 5=$ stimme absolut $\mathrm{zu}$ ); ${ }^{*}=\mathrm{p}<0,05 ;\left(^{*}\right)=\mathrm{p}<0,1$.

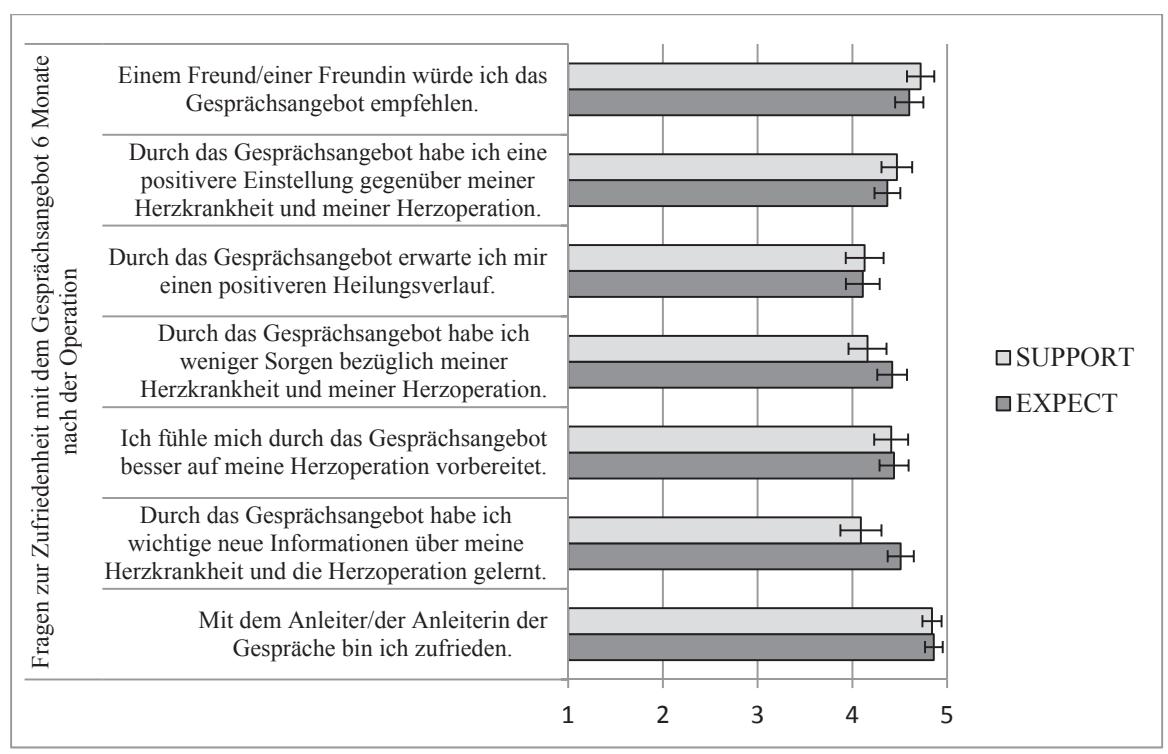

chen präoperativen psychologischen Interventionen (der Erwartungsintervention EXPECT oder der supportiven Therapie SUPPORT). Die Datenerhebung fand zwischen April 2011 und Mai 2015 zu 4 Zeitpunkten in der Klinik für Herzund Gefäßchirurgie am Universitätsklinikum Gießen und Marburg statt: zu Baseline (T0: etwa 7-10 Tage vor der Operation), nach der psychologischen Intervention (T1: Aufnahme des Patienten in das Krankenhaus; etwa 1 Tag vor der Operation), postoperativ (T2: etwa 6 Tage nach der Operation) und 6 Monate postoperativ (T3). Detaillierte Informationen zum Studiendesign sind im Studienprotokoll zu finden [Laferton et al., 2013]. Die Studie wurde von der Ethikkommission der Philipps-Universität Marburg genehmigt und entspricht den ethischen Standards der Deklaration von Helsinki in ihrer erweiterten Form von 1975 und ihren Zusätzen von 1983, 1989 und 1996.

\section{Ergebnisvariablen}

Die primäre Ergebnisvariable war die krankheitsbedingte Beeinträchtigung (Pain Disability Index) [Tait et al., 1990] der Patienten 6 Monate nach der Operation. Ergebnisse zum primären Outcome, zu weiteren klinischen Ergebnisvariablen (z.B. mentale und physische Lebensqualität, Angst, Depressivität, Erwartungen), zu biologischen Parametern (z.B. Interleukin-6 oder Adrenalin) [Rief et al., 2017; Salzmann et al., 2017] und zur Aufenthaltsdauer der Patienten im Krankenhaus wurden andernorts berichtet [Auer et al., 2017]. Die Zufriedenheit der Patienten mit der Intervention wurde zu 2 Zeitpunkten erfragt: direkt nach
Abschluss der Intervention (vor der Operation) und 6 Monate nach der Operation (Follow-up). Es wurden jeweils 7 Fragen gestellt, die die Patienten auf einer Skala von 1 bis $5(1=$ stimme absolut nicht $\mathrm{zu}, 2=$ stimme weniger $\mathrm{zu}, 3=$ weder noch, $4=$ stimme etwas zu, $5=$ stimme absolut zu) in einem Paper-Pencil-Fragebogen beantworten sollten (Abb. 1). Aus den Antworten der Patienten wurden Mittelwerte berechnet.

\section{Rekrutierung}

Patienten auf der Warteliste für eine aortokoronare Bypass-Operation mit oder ohne Herzklappenersatz der Klinik für Herz- und Gefäßchirurgie Gießen und Marburg wurden vor der Aufnahme in das Krankenhaus telefonisch kontaktiert. Die Studienteilnehmer mussten mindestens 18 Jahre alt und für eine erste elektive Bypass-Operation mit Unterstützung einer Herz-Lungen-Maschine vorgesehen sein. Eine schwerwiegende psychische oder eine lebensbedrohliche (nichtkardiale) Erkrankung sowie eine vorhergehende Bypass-Operation oder die Teilnahme an anderen Studien waren Ausschlusskriterien.

249 Patienten kamen für die Teilnahme an dieser Studie infrage. Davon lehnten 72 Personen $(28,9 \%)$ eine Teilnahme ab, da sie entweder kein Interesse an einer präoperativen psychologischen Intervention oder keine Möglichkeit hatten, für die Intervention in die Klinik zu kommen. Insgesamt wurden N = 124 Patienten konsekutiv in die Studie eingeschlossen, von denen 3 von der statistischen Analyse ausgeschlossen wurden. Ein Patient zog seine Einverständniser- 
klärung zur Studienteilnahme zurück, bei 1 Patienten stellte sich heraus, dass er keine Bypass-Operation benötigte, und 1 Patient wurde aufgrund einer sehr langen Zeit zwischen Intervention und Operation (mehr als 4 Wochen) ausgeschlossen. Eine detaillierte Übersicht über die Stichprobeneigenschaften ist in der Publikation zum primären Outcome zu finden [Rief et al., 2017]. Die Gesamtstichprobe war im Mittel 66,1 (Standardabweichung (SD) $=8,27$ ) Jahre alt $($ EXPECT $=66,3(\mathrm{SD}=7,88)$ Jahre; SUPPORT $=64,9(\mathrm{SD}=8,15)$ Jahre; $\mathrm{SMC}=$ $67,0(\mathrm{SD}=8,76)$ Jahre) und bestand mit $84,3 \%$ vorwiegend aus männlichen Teilnehmern (EXPECT $=84,6 \%$; SUPPORT $=81,6 \%$; SMC $=86,4 \%$ ).

\section{Ablauf und Assessment}

Mit Patienten, die Interesse an einer Studienteilnahme bekundeten, wurde ein erstes persönliches Treffen im Klinikum 7-10 Tage vor der Operation vereinbart, bei dem diese alle relevanten Informationen erhielten. Nach der schriftlichen Einwilligung in die Studienteilnahme fand die Ausgangsmessung statt. Klinische Psychologen mit fortgeschrittenen Kenntnissen in kognitiver Verhaltenstherapie führten das Strukturierte Klinische Interview nach DSM-IV durch, um psychische Komorbiditäten zu erfassen. Soziodemografische Daten wie Alter, Geschlecht und Bildung wurden erfragt, medizinische Daten wurden den Patientenakten entnommen. Nach der Ausgangsmessung wurden die Patienten randomisiert und 1 der 3 Studienbedingungen zugeteilt. Eine Studienassistenz in der Klinik unterstützte bei der Datenerhebung und kümmerte sich um organisatorische Aspekte. Die medizinischen Gespräche zur Vorbereitung auf die Operation fanden in der Regel am Aufnahmetag (1 Tag vor der Operation) statt und bestanden aus präoperativen Aufklärungsgesprächen mit einem Herzchirurgen und einem Anästhesisten. Dabei liegt der Fokus auf der Erläuterung der medizinischen Prozedur, der Aufklärung über medizinische Risiken und dem Unterzeichnen der Einverständniserklärung. Gespräche über die Erwartungen der Patienten, vor allem auch in Bezug auf die Zeit nach der Operation, sind nicht systematisch in diese präoperativen Aufklärungsgespräche eingebettet. Darüber hinaus werden die Patienten am Aufnahmetag auch von Mitarbeitern der Pflege empfangen und betreut.

\section{Rahmenbedingungen und Ablauf der psychologischen Kurzintervention}

Sowohl die erwartungsoptimierende als auch die supportive psychologische Intervention umfassten jeweils 2 individuelle Sitzungen (à $50 \mathrm{~min}$ ) vor Ort und 2 Telefongespräche (à $20 \mathrm{~min}$ ) zwischen der Ausgangsuntersuchung und der Operation (vgl. Online Supplemental Abb. 1., Online Supplemental Material; www.karger.com/?DOI=485430). Der erste persönliche Gesprächstermin fand etwa 7-10 Tage vor der Operation statt; dann wurden beide Telefonate mit den Patienten geführt; anschließend fand der letzte persönliche Gesprächstermin statt, zumeist am Tag der Aufnahme in die Klinik (etwa 1 Tag vor der Operation). Etwa 6 Wochen nach der Operation wurde ein weiteres 20-minütiges BoosterTelefonat durchgeführt. Die Gespräche wurden in einem Raum der Klinik für Herz- und Gefäßchirurgie am Universitätsklinikum Gießen und Marburg in Kooperation mit der Abteilung für Klinische Psychologie und Psychotherapie der Philipps-Universität Marburg durchgeführt.

Die Interventionen wurden von klinischen Psychologen mit fortgeschrittenen Kenntnissen in kognitiver Verhaltenstherapie durchgeführt; die Psychologen (1 Frau, 2 Männer) wurden in der Anwendung der manualisierten Intervention geschult und regelmäßig supervidiert (K.W.). Alle Therapiesitzungen wurden auf Video aufgezeichnet. Die Befolgung des Therapiemanuals für die unterschiedlichen Interventionen wurde durch verblindete Gutachter überprüft. Nach der letzten Sitzung wurden den Patienten die Aufnahmen aller Sitzungen auch als Audio-CD ausgehändigt. Darüber hinaus erhielten die Patienten in der EXPECT-Gruppe zu Beginn der Intervention eine «Herzfibel». Darin waren die wesentlichen Inhalte der Intervention in allgemein verständlicher Sprache sowie einiger Arbeitsblätter für Patienten zusammengefasst und mit Bildern anschaulich dargestellt. Die Patienten sollten die besprochenen Inhalte zwischen den Sitzungen mithilfe von Hausaufgaben wiederholen und verinnerlichen.

\section{Hintergründe der Entwicklung der Erwartungsintervention}

Eine kognitiv-behaviorale, vornehmlich psychoedukative Intervention wurde mit dem primären Ziel entwickelt, die Erwartungen von Patienten vor einer aortokoronaren Bypass-Operation an den Genesungsverlauf danach zu optimie- ren. Des Weiteren sollte die Intervention in den klinischen Alltag integrierbar sein. Die Intervention wurde auf der Basis des Common-Sense-Modells der Selbstregulation [Cameron und Leventhal, 2003; Leventhal et al., 1980] konzipiert. Zur Erarbeitung der Interventionsinhalte wurden ergänzend auch eigene qualitative Patienteninterviews sowie qualitative Arbeiten anderer Autoren [z.B. Lindsay et al., 2000] berücksichtigt, die Themen wie starke körperliche Einschränkung, damit einhergehende eingeschränkte Aktivität, Abhängigkeit von anderen, aber auch Angst vor dem Tod und Verlust der Kontrolle über die eigene Gesundheit besonders relevant erscheinen ließen. Das Common-Sense-Modell der Selbstregulation geht davon aus, dass Patienten über subjektive Krankheitsrepräsentationen («illness beliefs» oder «perceptions») verfügen, die beschreiben, wie ein Patient seine Krankheit versteht und erlebt [Cameron und Leventhal, 2003; Leventhal et al., 1980]. So entwickeln Patienten beispielsweise Annahmen darüber, wie ihre Krankheit entstanden ist (Ursache), welche Symptome ihre Krankheit hervorruft (Identität), wie lange sie noch anhalten wird (Zeitverlauf), welche Folgen bestimmte Symptome haben werden (Konsequenzen) und ob sie durch eigenes Handeln (persönliche Kontrolle) oder aufgrund einer Behandlung (Behandlungskontrolle) eine Besserung erfahren werden. Erwartungen sind ein inhärenter (wenn auch nicht expliziter) Bestandteil dieser Krankheitsrepräsentationen [Cameron und Leventhal, 2003], da sie in der Regel Annahmen über das Auftreten von Ereignissen oder Wahrnehmungen in der Zukunft darstellen [Laferton et al., 2017]. Funktionale Behandlungserwartungen von Patienten sind mit verbesserten Behandlungsergebnissen assoziiert [Cameron und Leventhal, 2003]. Darüber hinaus ist es diesem Modell nach sinnvoll, maladaptive Annahmen bzw. Erwartungen der Patienten zu thematisieren und gegebenenfalls zu modifizieren.

Das übergeordnete Ziel der Intervention bestand darin, dass die Patienten der aortokoronaren Bypass-Operation, deren Ergebnis und der darauffolgenden Zeit mit größtmöglicher Zuversicht und gleichermaßen realistischen sowie individualisierten und funktionalen Erwartungen begegnen. Um die Vielfalt der in bisherigen Studien erfassten Patientenerwartungen zu integrieren und einer Schwierigkeit der bisherigen Erwartungsforschung - der Heterogenität der erfassten Konstrukte sowie deren Messung - entgegenzuwirken, haben Laferton et al. [2017] ein Integratives Modell der Erwartungen von Patienten, die sich einer medizinischen Behandlung unterziehen, vorgeschlagen. Auch wenn das Modell erst nach der Konzeption der Intervention publiziert wurde, nehmen wir im Folgenden Bezug darauf, da die dort integrierten Erwartungskonzepte für das Verständnis unserer Intervention hilfreich sind. An diesem Modell sollen sich die Bezeichnungen der unterschiedlichen Erwartungskonstrukte im Folgenden orientieren. Erwartungen werden hier als subjektive probabilistische Zukunftsaussagen über das Auftreten von Ereignissen konzipiert und dabei vor allem in Abgrenzung zu wertebasierten Konstrukten wie Wünschen oder Hoffnungen verstanden. Ausgehend vom Integrativen Modell der Erwartungen [Laferton et al., 2017] sollten die Patienten ein besseres Verständnis ihrer Erkrankung erlangen und eine positive Ergebniserwartung (outcome expectation) hinsichtlich der bevorstehenden Operation ausbilden (z.B. eine geringere krankheitsbedingte Beeinträchtigung). Diese Ergebniserwartungen setzen sich nach dem Integrativen Modell aus Erwartungen zusammen, die ein Patient hinsichtlich seines eigenen krankheits- oder behandlungsbezogenen Verhaltens (behavior-related expectations) hat, sowie durch die Erwartungen, die er hinsichtlich seiner Behandlung (treatment-related expectations) ausbildet. Bei den verhaltensbezogenen Erwartungen unterscheidet das Modell noch zwischen Selbstwirksamkeit (self-efficacy) und Verhaltensergebniserwartung (behavior outcome expectation), die gemeinsam als persönliche Kontrollerwartung (personalized outcome expectancy / personal control belief) bezeichnet werden. Ein Patient mit hoher Selbstwirksamkeit wird sich nicht zwangsläufig sportlich betätigen, solange er nicht auch im Sinne einer positiven Verhaltensergebniserwartung davon ausgeht, dass sich dieses Verhalten positiv auf seine Gesundheit auswirkt. Sowohl verhaltensbezogene als auch behandlungsbezogene Ergebniserwartungen können darüber hinaus hinsichtlich der erwarteten positiven Aspekte (benefits) oder Nebenwirkungen (side effects) differenziert sowie in Bezug auf Prozesse innerhalb des Patienten (z.B. Erwartungen hinsichtlich des Auftretens von Symptomen) oder externale Faktoren (z.B. Reaktion anderer im sozialen Umfeld) weiter unterschieden werden. 
Um die Patienten dabei zu unterstützen, eine generell positive Ergebniserwartung auszubilden, sollten vor allem Erwartungen hinsichtlich der persönlichen Kontrollmöglichkeiten des Heilungsverlaufs und im Hinblick auf die positiven Konsequenzen der Behandlung gefördert werden. Die Erwartungen der Patienten hinsichtlich Nebenwirkungen der Operation sollten ebenfalls durch adäquate Informationen optimiert werden, wobei Fehlannahmen (z.B. «Mein Herz ist kaputt») korrigiert wurden. Auch eine Steigerung der Coping-Möglichkeiten (task and coping self-efficacy) war ein Ziel der Intervention.

Der Ablauf sollte im Hinblick auf die Struktur und übergeordnete Themen für alle Patienten identisch sein, wobei eine individuelle Schwerpunktsetzung möglich sein sollte. Darüber hinaus wurde die Integration der Partner berücksichtigt. Da auch die Erwartungen der Partner eine wichtige Rolle für die Patienten spielen [Figueiras und Weinman, 2003; Weinman et al., 2000; Broadbent et al., 2009b], wurden diese eingeladen, an der Intervention teilzunehmen, wenn die Patienten einverstanden waren.

Im Folgenden werden die Inhalte der einzelnen Sitzungen der EXPECTIntervention genauer beschrieben (für einen Überblick siehe Online Supplemental Tab. 1., Online Supplemental Material; www.karger.com/?DOI=485430).

Sitzung 1 (7-10 Tage vor der Operation, Dauer: 45-60 min):

«Die Bypass-Operation - neu gewonnene Freiheit und Lebensretter»

$\mathrm{Zu}$ Beginn der Sitzung wurde der Patient begrüßt und über das Vorgehen, den zeitlichen Ablauf und die Ziele der Interventionen informiert, bevor ihm die Herzfibel ausgehändigt wurde. Er erhielt darüber hinaus die Information, dass die Einstellungen und Erwartungen eines Patienten für die Genesung eine wichtige Rolle spielen. Im Fokus der ersten Sitzung standen das subjektive Krankheitsmodell des Patienten und die anstehende Bypass-Operation. Ausgehend von seinem subjektiven Krankheits-/Behandlungsmodell wurde mit dem Patienten ein grundlegendes Verständnis seiner Erkrankung, der damit assoziierten Symptome und des medizinischen Eingriffs erarbeitet. Um eine möglichst hohe und positive behandlungsbezogene Ergebniserwartung aufzubauen, sollte dem Patienten vermittelt werden, dass der Eingriff eine Heilung der gegenwärtig belastenden und einschränkenden Umstände der Herzerkrankung bedeutet. Dabei sollte vor allem verdeutlicht werden, dass die anstehende Operation die Unterversorgung des Herzens mit Sauerstoff aufheben, damit assoziierte Symptome (z.B. Enge in der Brust) verschwinden und die körperliche Leistungsfähigkeit wieder ansteigen würde. Durch die Besprechung des Ablaufs der Operation und der Zeit danach wurden strukturelle bzw. Prozesserwartungen (structural/process expectations) thematisiert. Diese stellen einen wichtigen Aspekt des Behandlungskontextes dar; so haben Herzpatienten beispielsweise umso positivere Ergebniserwartungen, je invasiver die angewendeten Methoden sind [Hirani et al., 2008]. Es wurde darauf geachtet, dass die Informationen leicht verständlich formuliert wurden (Stellen Sie sich einen Motor vor, der eigentlich noch gut läuft, aber kein Benzin mehr erhält, weil die Benzinleitung verstopft ist. Durch eine neue Benzinleitung erhält der Motor wieder ausreichend Treibstoff und kann wieder ganz normal funktionieren. So ähnlich ist es mit Ihrem Herzen. Damit Ihr Herz wieder optimal arbeiten kann, wird eine neue Leitung gelegt, um das Herz wieder ausreichend mit Sauerstoff zu versorgen.). Darüber hinaus wurden falsche Vorstellungen und Annahmen (z.B. «Mein Herz ist kaputt und wird nie wieder richtig funktionieren») korrigiert.

Die Therapeuten betonten, dass die Operation ein oft durchgeführtes und damit sehr sicheres Verfahren ist. Auch die Kompetenz der Chirurgen und des gesamten Krankenhauspersonals wurde positiv beschrieben (Das sind Profis. Die machen das täglich und haben sehr viel Erfahrung.). Positive Ergebniserwartungen wurden vor allem durch Informationen über Symptomverbesserungen, eine Steigerung der Lebensqualität und einen positiv-realistischen Erholungsprozess gefördert, indem auch explizit Erwartungen hinsichtlich des zeitlichen Verlaufs (timeline expectations) thematisiert wurden. Um die Informationen für den Patienten verständlich zu gestalten und die Übertragung auf seine individuelle Situation zu fördern, sollten die Vorteile der Bypass-Operation auch in eine Antizipation der Zeit nach der Operation einfließen. Unter Berücksichtigung von Expertenempfehlungen der Society of Thoracic Surgeons [2009] wurde dabei gemeinsam mit dem Patienten ein gestufter Aktivitätsplan (Meine Aktivitäten nach der Herzoperation) entwickelt, in dem der Patient Aktivitäten beschrei- ben sollte, die er in den ersten 6 Wochen (z.B. leichte Gartenarbeit wie Blumen umtopfen), frühestens 6 Wochen nach der Operation (z.B. etwas intensivere Gartenarbeit wie Rasenmähen) und 3 Monate nach der Operation (z.B. sehr intensive Gartenarbeit wie Holz hacken) wieder ausführen mochte (für Beispiele siehe Online Supplemental Tab. 2., Online Supplemental Material; www.karger. com/?DOI=485430).

Von diesen Überlegungen ausgehend wurde der Patient unter Einbezug seiner persönlichen Interessen und Wünsche zu einer Imaginationsübung angeleitet, indem er sich eine Situation 6 Monate nach einer erfolgreich verlaufenen Operation vorstellen sollte, die den Erfolg und die gesteigerte Lebensqualität nach dem Eingriff ausdrückt. Der Patient wurde im Rahmen der Imagination ermutigt, sich die Zeit nach der Operation vorzustellen, wobei er neben den für ihn persönlich wichtigen Ereignissen an die damit assoziierten Emotionen denken sollte. Diese Imagination wurde gemeinsam ausgestaltet, indem vor allem auch Sinneseindrücke und positive Emotionen elaboriert wurden (für ein Patientenbeispiel siehe Online Supplemental Kasten 1., Online Supplemental Material; www.karger.com/?DOI=485430). Der Patient wurde dazu ermutigt, diese Übung so oft wie möglich durchzuführen.

Zusammenfassend sollte der Patient im Hinblick auf sein subjektives Krankheitsmodell in positiven und adaptiven behandlungsbezogenen Ergebniserwartungen bestärkt werden; maladaptive Nebenwirkungserwartungen der Behandlung sollten hinterfragt und verändert werden. Als Kernbotschaft sollte der Patient aus dieser ersten Sitzung mitnehmen, dass die Herzoperation eine sehr gute Aussicht auf Erfolg bietet und sich seine Lebensqualität postoperativ langfristig verbessern wird. Zum Ende der Sitzung wurden das Erarbeitete unter Betonung der Vorteile der Operation zusammengefasst und ein Ausblick auf die kommenden Termine gegeben. Zur Festigung des Besprochenen wurde dem Patienten als Hausaufgabe aufgetragen, den entsprechenden Abschnitt in der Herzfibel nochmals zu lesen.

Telefonat 1 (etwa 5 Tage vor der Operation, Dauer: $20 \mathrm{~min}$ ):

«Die Bypass-Operation und mein Gesundheitsverhalten - Chance zum Neuanfang I»

$\mathrm{Zu}$ Beginn des Telefonats wurden die Inhalte der letzten Sitzung kurz wiederholt. Anschließend wurde zum aktuellen Thema, der Steigerung der verhaltensbezogenen Ergebniserwartungen, übergeleitet. Um die Ergebniserwartungen im Hinblick auf das eigene Verhalten zu optimieren, wurden zunächst die subjektiven Ursachen für die Herzerkrankung des Patienten erhoben und diese dann durch psychoedukative Elemente zu Ursachen und Risikofaktoren ergänzt. Hierbei wurde der Fokus vor allem auf Lebensgewohnheiten (z.B. Rauchen, Ernährung, Sport) und auf Aspekte gelegt, auf die der Patient selbst Einfluss hat, deren Veränderung einen positiven Effekt auf die Gesundheit hätte (verhaltensbezogene Ergebniserwartungen) und deren Umsetzung er sich zutraut (Selbstwirksamkeitserwartung). Insbesondere die Zusammenhänge zwischen den Lebensgewohnheiten und der Herzkrankheit wurden diskutiert, um für jeden $\mathrm{Pa}$ tienten herauszufinden, wie er nach überstandener Operation ganz konkret Kontrolle über das Fortschreiten bzw. das erneute Auftreten seiner Erkrankung ausüben könne. Diese neu gewonnene Kontrolle sollte gegenüber der aktuellen Situation vor der Operation als Vorteil herausgestellt werden, indem sie als «Chance zum Neuanfang» bezeichnet wurde. Der Therapeut unterstützte den Patienten dabei, Annahmen wie «Ich habe keine Kontrolle über meine Herzkrankheit» in Richtung «Ich habe die Möglichkeit meine Herzerkrankung positiv zu beeinflussen» zu verändern. Abschließend wurden die Inhalte der Sitzung rekapituliert, die Imaginationsübung unter Einbezug der neu erarbeiteten Inhalte durchgeführt und ein Ausblick auf das nächste Telefonat gegeben. Der Patient erhielt die Hausaufgabe, zu überlegen, wo und wie er seine Herzerkrankung zukünftig selbst positiv beeinflussen könne.

Telefonat 2 (etwa 3 Tage vor der Operation, Dauer: $20 \mathrm{~min}$ ):

«Die Bypass-Operation und mein Gesundheitsverhalten - Chance zum Neuanfang II»

Auch das zweite Telefonat begann mit der Wiederholung der Inhalte des letzten Kontakts. Ausgehend von der erneuten Betonung der Bedeutung des eigenen Gesundheitsverhaltens für den zukünftigen Gesundheitszustand wurde 
an die persönlichen Risikofaktoren der Patienten angeknüpft. Auf der Basis der identifizierten persönlichen Risikofaktoren im Gesundheitsverhalten der Patienten wurde ein Gesundheitsvertrag «ausgehandelt», in dem sich der Patient auf konkrete Schritte festlegen sollte, um seine Risikofaktoren zukünftig selbst positiv zu beeinflussen. Dabei wurde gemeinsam mit dem Patienten darüber reflektiert, welche konkreten Veränderungen er in seinem Leben vornehmen möchte. Um die Motivation zu steigern, wurde auch der Grund für eine Veränderung (z.B. «Welche Vorteile hätte es, wenn Sie sich gesünder ernähren würden?») jeweils identifiziert und festgehalten (z.B. «Ich will mich gesünder ernähren, um die Verengung meiner Arterien zu verhindern»). Selbstwirksamkeitserwartungen wurden gesteigert, indem zukünftige gesunde Verhaltensweisen wie Sport oder eine ausgewogene Ernährung möglichst konkret im Gesundheitsvertrag festgehalten wurden (z.B. « $2 \times$ pro Woche Fisch essen»). Dabei achtete der Therapeut darauf, dass nur solche Verhaltensweisen notiert wurden, die sich der Patient auch wirklich zutraute. Darüber hinaus wurde dem Patienten empfohlen, den Vertrag zu unterschreiben und für Angehörige gut sichtbar aufzuhängen, um das Commitment zu steigern. Nach einer Wiederholung der Inhalte und einer Durchführung der Imaginationsübung erhielten die Patienten einen Ausblick auf die nächste Sitzung. Als Hausaufgabe sollte der Gesundheitsvertrag fertiggestellt werden.

Sitzung 2 (1 Tag vor der Operation, Dauer: 45-60 min):

«Nebenwirkungen der Bypass-Operation - ich bin gewappnet»

Nach einer Wiederholung der bisherigen Inhalte wurde der Schwerpunkt in der zweiten persönlichen Sitzung auf erwartete Nebenwirkungen durch die Behandlung und die Vorbereitung auf bzw. den Umgang mit unangenehmen, aber nicht bedrohlichen, «normalen» Symptomen nach der Operation gelegt. Hierbei wurden vor allem negative Behandlungsergebniserwartungen sowie die Selbstwirksamkeitserwartung hinsichtlich der eigenen Coping-Möglichkeiten bearbeitet, um den Patienten in die Lage zu versetzen, bestmöglich mit unangenehmen Symptomen umzugehen. Diese Sitzung fand am Tag der Aufnahme in das Krankenhaus statt und sollte neben den thematischen Inhalten auch stabilisierend wirken, da die Patienten aufgrund der zeitlichen Nähe zur Operation und des zumeist anstrengenden Prozedere am Aufnahmetag oft ein erhöhtes An spannungsniveau aufwiesen. Der Patient sollte zudem eine realistische Erwartung hinsichtlich unangenehmer, aber nicht bedrohlicher Symptome und Empfindungen nach der Operation erhalten. Dadurch sollte einer unangemessenen Katastrophisierung (und einer damit einhergehenden negativen Erwartungshaltung) unangenehmer Begleiterscheinungen der Operation vorgebeugt werden. So wurde beispielsweise über das Aufwachen nach der Operation auf der Intensivstation gesprochen. Hierbei wurden die zu erwartenden Schläuche, Geräusche und Symptome detailliert besprochen und deren Funktion bzw. Ursache erläutert. Darüber hinaus wurden von Patienten geäußerte Ängste (z.B. vor möglichen Komplikationen) validiert und normalisiert. In Bezug auf angemessene Er wartungen hinsichtlich auftretender Symptome in der Erholungsphase nach der Operation (z.B. Schmerzen) wurden Coping-Strategien gesammelt, um auch hier die persönlichen Kontrollerwartungen zu steigern. Das Ergebnis wurde in einem persönlichen Problemlöseschema (Werkzeugkoffer gegen unangenehme Empfindungen) festgehalten, in dem der Patient seine individuellen Bewältigungsstrategien für erwartete Probleme (z.B. bei Schmerzen nach Schmerzmitteln fragen) notierte. Zum Abschluss der letzten Sitzung wurden die Vorteile durch die Operation im Vergleich zur Ausgangssituation im Sinne einer optimierten behandlungsbezogenen Ergebniserwartung nochmals herausgestellt und die wiedererlangte Kontrolle über die Herzkrankheit im Sinne einer verhaltensbezogenen Ergebniserwartung betont. Nach einer Zusammenfassung der wesentlichen Interventionsinhalte und einer Durchführung der Imagination wurden wiederum die Inhalte zusammengefasst, die Audio-CD übergeben, offene Fragen geklärt und sich verabschiedet.

Die Patienten hatten am Ende dieser Sitzung die Möglichkeit, einen «Brief an mich selbst» zu schreiben, in dem sie die für sie wichtigsten Aspekte aus der Intervention als Hausaufgabe festhalten konnten. Dieser Brief wurde an das Studienteam übergeben und dem Patienten etwa 6 Wochen nach der Operation zugesendet, um ihn an die wichtigsten Aspekte der Intervention in seinen Worten zu erinnern.
Booster-Telefonat (etwa 6 Wochen nach der Operation, Dauer: 20 min)

$\mathrm{Zu}$ Beginn des Booster-Telefonats wurden die Patienten nach ihrem derzeitigen Befinden und dem Verlauf nach der Operation gefragt. Anschließend wurde die Umsetzung der Interventionsinhalte bzw. der Umsetzungserfolg der geplanten Ziele (Aktivitäten nach der Herzoperation aus Sitzung 1; Gesundheitsvertrag aus Telefonat 2; Werkzeugkoffer gegen unangenehme Empfindungen aus Sitzung 2) thematisiert. Je nach Erfolg wurden die Patienten in ihrer bisherigen Umsetzung bestärkt oder - bei Schwierigkeiten in der Umsetzung - noch hinsichtlich der geplanten Maßnahmen befragt und die dahinterstehende Motivation reflektiert. Der Therapeut half dabei, Lösungen für bestehende Barrieren oder eine Adaptation des Plans zu entwickeln, indem er sowohl die verhaltensbezogenen Ergebniserwartungen als auch die Selbstwirksamkeitserwartungen thematisierte. Darüber hinaus wurde die Imaginationsübung unter Betonung der Handlungspläne als Weg zu dem vorgestellten Zielzustand durchgeführt. Am Ende des Telefonats wurden nochmals die Bedeutung der Handlungspläne für die Gesundheit des Patienten hervorgehoben, auf den weiteren Ablauf (Follow-up-Messung) verwiesen und dem Patienten eine gute Genesung gewünscht.

\section{Supportive Intervention}

Um die Spezifizität der erwartungsoptimierenden Intervention zu testen, erhielten die Patienten in der supportiven Therapie dasselbe Ausmaß an therapeutischer Aufmerksamkeit (identische Anzahl an Sitzungen mit identischer Länge), ohne explizit an Erwartungen zu arbeiten. Diese Intervention legte einen stärkeren Fokus auf eine gute therapeutische Interaktion zwischen Patient und Behandler und hatte vor allem die Anwendung allgemeiner Wirkfaktoren psychotherapeutischer Interventionen zum Ziel: So hörten die Therapeuten empathisch zu, validierten die Empfindungen und Gedanken der Patienten und halfen dabei, Emotionen zu benennen und diese zu erleben. Die Patienten in dieser Gruppe hatten die Möglichkeit, frei zu entscheiden, über welche Themen sie sprechen mochten. Diese Art der supportiven Therapie wurde bereits in früheren Studien angewendet [Cohen et al., 2011].

\section{Statistische Auswertung}

Da die Daten zur Zufriedenheit nicht normalverteilt waren, wurde ein Mann-Whitney-U-Test für jedes Item durchgeführt, bei dem die beiden psychologischen Interventionen miteinander verglichen wurden.

\section{Ergebnisse}

Insgesamt lag der Mittelwert aller Items, die zur Evaluation der psychologischen Intervention aus Patientensicht eingesetzt wurden, sowohl direkt nach der Intervention (jedoch vor der Operation) als auch 6 Monate nach der Operation im hohen bis sehr hohen Bereich (Abb. 1). Der Anteil der Patienten, die mit der Intervention nicht zufrieden waren (entspricht den Antworten $1=$ «stimme absolut nicht zu» oder 2 = «stimme weniger zu»), variierte für die supportive Gruppe zwischen 0 und 10,5\% (entspricht 0 bis 4 von 38 Patienten) und für die EXPECT-Gruppe zwischen 0 und $5,1 \%$ ( 0 bis 2 von 39 Patienten) pro Item direkt nach der Intervention. Sechs Monate nach der Intervention betrug dieser Anteil 2,6 bis $13,2 \%$ ( 1 bis 5 von 38 Patienten) in der supportiven Gruppe und 2,6 bis 5,1\% ( 1 bis 2 von 39 Patienten) in der Erwartungsintervention.

Direkt nach der Intervention hatten die Patienten, die EXPECT erhielten, eher den Eindruck, neue und wichtige Informationen zu ihrer Krankheit und zur Operation zu erhalten $(U=391 ; \mathrm{p}=0,021$; $r=0,28$ (Abb. 1)), und erwarteten einen positiveren Heilungsverlauf $(\mathrm{U}=420 ; \mathrm{p}=0,049 ; \mathrm{r}=0,24)$ als die Patienten, die die supportive Intervention erhielten. Ein Trend, jedoch kein signifikantes 
Ergebnis, zeigte sich im Hinblick darauf, dass sich die Patienten nach der Erwartungsintervention eher gut auf die Operation vorbereitet fühlten als Patienten in der supportiven Gruppe ( $\mathrm{U}=$ 439,$5 ; \mathrm{p}=0,072$ ). Hinsichtlich aller anderen Items zeigten sich präoperativ keine signifikanten Unterschiede zwischen beiden Interventionsgruppen (alle $p \geq 0,462$ ); sechs Monate nach der Intervention unterschieden sich die Gruppen ebenfalls bei keinem Item (alle $\mathrm{p} \geq 0,125$ ).

\section{Diskussion}

Im Rahmen der PSY-HEART-Studie (Psychologische Intervention für Herzpatienten) wurde eine präoperative psychologische Kurzintervention mit dem primären Ziel entwickelt, Patientenerwartungen zu optimieren. Ein weiteres wichtiges Kriterium war die Sicherstellung der Durchführbarkeit auf einer herzchirurgischen Station. Die neu entwickelte erwartungsoptimierende Intervention führte 6 Monate nach der Operation zu besseren Ergebnissen verglichen mit Patienten, die nur eine medizinische Standardbehandlung erhielten [Rief et al., 2017]. In dem vorliegenden Artikel wurden die Inhalte der erwartungsoptimierenden Intervention genauer beschrieben und die Zufriedenheit der Patienten mit den präoperativen psychologischen Interventionen dargestellt. In der ersten persönlichen Sitzung wurde primär an einer erhöhten behandlungsbezogenen Ergebniserwartung hinsichtlich der Vorteile einer Operation und eines besseren Verständnisses der eigenen Erkrankung gearbeitet, während die beiden anschließenden Telefonate auf eine verbesserte verhaltensbezogene Ergebniserwartung fokussierten. Im letzten persönlichen Kontakt wurden Fehlannahmen korrigiert und die Patienten auf den optimalen Umgang mit Nebenwirkungen (behandlungsbezogene Ergebniserwartung und Selbstwirksamkeit hinsichtlich der Coping-Möglichkeiten) der Operation vorbereitet. Durchgehend wurde mithilfe einer Imaginationsübung daran gearbeitet, ein positives Zukunftsbild und somit positive Ergebniserwartungen hinsichtlich der langfristigen Konsequenzen der Operation aufzubauen.

Die EXPECT-Intervention wurde auf der Basis jüngerer Erkenntnisse aus der Placeboforschung entwickelt, wonach präoperative Erwartungen signifikante Prädiktoren für postoperative Ergebnisse sind [Auer et al., 2016; Juergens et al., 2010; Schedlowski et al., 2015] und Erwartungen in der Behandlung sowohl körperlicher als auch psychischer Erkrankungen eine immer wichtigere Rolle spielen [Rief und Glombiewski, 2016; Schedlowski et al., 2015]. Mithilfe der erwartungsoptimierenden Intervention konnte beispielsweise die verhaltensbezogene Ergebniserwartung (persönliche Kontrollerwartungen) der Patienten signifikant gesteigert werden [Rief et al., 2017]. Höhere persönliche Kontrollerwartungen gehen mit einer gesteigerten Lebensqualität und geringeren Depressivität nach Bypass-Operationen einher [Kidd et al., 2016].

Obwohl die Zufriedenheit der Patienten für beide dargestellten präoperativen Interventionen als hoch eingeschätzt werden kann, war die erwartungsoptimierende der supportiven Intervention punktuell überlegen. So fühlten sich die Patienten in der EXPECT-
Bedingung noch etwas besser auf die Operation vorbereitet. Patienten, die vor einer aortokoronaren Bypass-Operation stehen, scheinen sich generell zusätzliche präoperative Gespräche zu wünschen. Es gibt Hinweise darauf, dass in Aufklärungsgesprächen vor herzchirurgischen Eingriffen wichtige Information hinsichtlich der $\mathrm{zu}$ erwartenden Konsequenzen für die Lebensqualität fehlen [Hauptman et al., 2013; Pedersen et al., 2017]. Die von uns entwickelte erwartungsoptimierende Intervention könnte neben einer Erwartungsoptimierung eine solche unzureichende Kommunikation zumindest teilweise ausgleichen.

Allerdings ist $\mathrm{zu}$ erwähnen, dass einige wenige Patienten mit der Intervention nicht zufrieden waren. Auch wenn die Anzahl dieser Patienten sehr gering sein mag, scheint dies jedoch darauf hinzudeuten, dass weiterer Forschungsbedarf dahingehend besteht, jedem Patienten die jeweils beste Behandlung/Intervention zukommen zu lassen. Möglicherweise haben die psychoedukativen Inhalte vereinzelt auch zusätzliche Ängste oder mehr Stress induziert. So war beispielsweise einem Teilnehmer nicht bewusst, dass sein Brustkorb für die Operation geöffnet werden würde, was ihm sichtbar Angst bereitete. Diesbezüglich sollten in zukünftigen Studien individuelle Bewältigungsdispositionen stärker berücksichtigt werden, indem die Interventionen darauf abgestimmt werden, ob die untersuchten Personen eher dazu tendieren, Bedrohungen kognitiv zu vermeiden oder ihre Angst mithilfe weiterer Informationen zu bewältigen [Krohne und El-Giamal, 2008].

Auch wenn die erwartungsoptimierende Intervention in bestimmten Bereichen noch etwas bessere Ergebnisse gezeigt hat, wurde auch die supportive Intervention von den Patienten gut angenommen und führte ebenfalls zu einer signifikanten Verbesserung der krankheitsbedingten Beeinträchtigung. Für die medizinische Standardbehandlung galt dies hingegen nicht. Eine gute Interaktion zwischen Patient und Behandler gilt ebenfalls als wichtiger Placebomechanismus [Schedlowski et al., 2015]. Die Überlegenheit der EXPECT-Intervention gegenüber der medizinischen Standardbehandlung könnte auf die Kombination zweier gut abgesicherter Placebomechanismen - Erwartungen und eine gute Patient/Behandler-Interaktion - zurückzuführen sein. Inwiefern die erwartungsoptimierende tatsächlich der supportiven Intervention überlegen ist, muss in zukünftigen Studien überprüft werden.

Die Optimierung ungünstiger Ausgangserwartungen erscheint bei der Therapie psychischer Störungen sinnvoll, da Erwartungen auch in der Psychotherapie eine zentrale Rolle spielen und nahezu alle Vorgänge im Rahmen einer Psychotherapie, z.B. die Erklärung eines Störungsmodells durch den Therapeuten, Patientenerwartungen beeinflussen [Wampold, 2015; Rief und Glombiewski, 2016]. Erwartungen sagen darüber hinaus Behandlungsergebnisse vorher und sind ein guter Prädiktor für die Adhärenz bzw. den Abbruch der Therapie durch den Patienten [Constantino et al., 2011]. Ungünstige Erwartungen können bei Psychotherapien sogar zu negativen Effekten beitragen [Ladwig et al., 2014]. Da Erwartungen zentral für die Entstehung und Aufrechterhaltung von psychischen Störungen sind, sollte eine effektive Therapie zu einer langfristigen Modifikation störungsrelevanter Erwartungen führen [Rief und Glombiewski, 2016]. Obwohl einige psychologische Interventionen 
bereits direkt oder indirekt Patientenerwartungen optimieren, könnte der Erfolg psychotherapeutischer Behandlungen weiter gesteigert werden, wenn die Modifikation von Erwartungen noch stärker im Fokus stünde [Rief und Glombiewski, 2016].

Die große Stärke der beiden präoperativen psychologischen Interventionen ist die Durchführbarkeit im klinischen Setting auf einer herzchirurgischen Station. Allerdings wird (geschultes) Personal für die Durchführung einer solchen Intervention benötigt, was auf den ersten Blick mit zusätzlichen Kosten verbunden ist. Berücksichtigt man jedoch, dass mit der hier vorgestellten Intervention die Liegezeiten insgesamt verkürzt werden konnten [Auer et al., 2017], wird deutlich, dass mit präoperativen psychologischen Interventionen neben einer Verbesserung der langfristigen Operationsergebnisse Kosten eingespart werden können.

Die ersten Ergebnisse zu der neu entwickelten präoperativen psychologischen Intervention sind sehr vielversprechend; dies sollte jedoch durch multizentrische Studien abgesichert werden. Sofern diese die positiven Ereignisse aus der ersten Studie [Rief et al., 2017] bestätigen, sollten präoperative psychologische Interventionen für alle Patienten angeboten werden, die vor einer aortokoronaren Bypass-Operation stehen.

\section{Limitationen}

Trotz der positiven Studienergebnisse und der durchweg hohen Patientenzufriedenheit bleiben die Wirkmechanismen noch offen, d.h. diejenigen Aspekte der Intervention, die tatsächlich für die positiven Effekte ausschlaggebend sind. Da die Intervention zur Erwartungsoptimierung verschiedene Bausteine beinhaltet, die aus Ansätzen für das Selbstmanagement von chronischen Erkrankungen bereits bekannt sind, sollte kritisch überprüft werden, ob die reine Arbeit an Erwartungen für positive Effekte ausreicht. Allerdings erscheint eine genaue Abgrenzung zwischen einzelnen Konstrukten hier schwierig, da Erwartungen einen so zentralen Stellenwert für menschliches Erleben und Verhalten haben. Auch sollte genauer untersucht werden, welche Erwartungen den größten Beitrag zu einem verbesserten Ergebnis nach einer Bypass-Operation leisten, um Interventionen noch weiter verkürzen bzw. noch effektiver gestalten zu können. Trotz einer wachsenden Zahl an Studien, die die Wichtigkeit von Erwartungen im Zusammenhang mit verschiedenen medizinischen Problemen untersucht haben, ist es schwierig, die einzelnen Ergebnisse zusammenzufassen, da die Konzeptualisierung von Erwartungen und deren Erfassung sehr heterogen sind [Laferton et al., 2017]. Einen Ansatz für eine Integration bisheriger Forschungsergebnisse bietet das Integrative Erwartungsmodell von Laferton et al. [2017], an dem sich dieser Artikel im Hinblick auf die Bezeichnung der Erwartungskonstrukte bereits orientiert.

Etwa ein Drittel der für eine Studienteilnahme infrage kommenden Patienten lehnte eine Teilnahme ab, da sie entweder kein Interesse an einer präoperativen psychologischen Intervention oder keine Möglichkeit hatten, für die präoperative Intervention in die Klinik zu kommen. Ein möglicher Selektionsbias kann somit nicht ausgeschlossen werden und könnte zu einer Überschätzung der Zufriedenheitsergebnisse beigetragen haben, da wahrscheinlich eher Personen an der Studie teilnahmen, die einer präoperativen psychologischen Intervention gegenüber grundsätzlich aufgeschlossen waren. Hier könnte es einerseits sinnvoll sein, Interventionen zu entwickeln, die noch stärker ortsunabhängig per Telefon oder Internet durchgeführt werden können. Andererseits sollte noch genauer untersucht werden, weshalb Patienten solche präoperativen Interventionen ablehnen.

\section{Schlussfolgerungen}

In dem vorliegenden Artikel konnte gezeigt werden, dass eine präoperative psychologische Intervention, die auf die Nutzung von Mechanismen des Placeboeffekts in Form von Patientenerwartungen fokussiert, Ergebnisse herzchirurgischer Operationen verbessern kann [Rief et al., 2017] und mit einer hohen Patientenzufriedenheit einhergeht. Patienten, die vor einer aortokoronaren Bypass-Operation stehen, sollten die Möglichkeit haben, eine psychologische Intervention zur Erwartungsoptimierung in Anspruch zu nehmen. Patientenerwartungen und das Potenzial ihrer Optimierung durch geeignete Interventionen sollten auch in anderen medizinischen Anwendungsbereichen noch genauer untersucht werden, um die Versorgung von Patienten weiter zu verbessern.

\section{Hinweis}

Die Unterlagen sowie das Behandlungsmanual können bei Herrn Stefan Salzmann (stefan.salzmann@uni-marburg.de) angefordert werden.

\section{Danksagung}

Ein Dank geht an Frau Heike Hoffmann und Frau Prof. Dr. Pankuweit und ihr Team sowie an alle Mitarbeiter der herzchirurgischen Station in der Klinik für Herzchirurgie Marburg.

\section{Online Supplemental Material}

Online Supplemental Material To access the supplemental material, please refer to $w w w$.karger.com/?DOI=485430.

\section{Disclosure Statement}

Die Autoren erklären, dass kein Interessenkonflikt besteht, der die hier dargestellten Inhalte beeinflusst hat. Die Studie wurde unterstützt durch Projektmittel der Deutschen Forschungsgemeinschaft (Ri 574/21-1) im Rahmen der Forschergruppe 1328. 


\section{Literatur}

- Auer CJ, Glombiewski JA, Doering BK, Winkler A, Laferton JAC, et al: Patients' expectations predict surgery outcomes: a meta-analysis. Int J Behav Med 2016;23: 49-62.

- Auer CJ, Laferton JAC, Shedden-Mora MC, Salzmann S, Moosdorf RG, Rief W: Optimizing preoperative expectations leads to a shorter length of hospital stay in CABG patients: further results of the randomized controlled PSY-HEART trial. J Psychosom Res 2017;97: 82-89.

Barefoot JC, Brummett BH, Williams RB, Siegler IC, Helms MJ, et al: Recovery expectations and long-term prognosis of patients with coronary heart disease. Arch Intern Med 2011;171:929-935.

Broadbent E, Ellis CJ, Thomas J, Gamble G, Petrie KJ: Further development of an illness perception intervention for myocardial infarction patients: a randomized controlled trial. J Psychosom Res 2009a;67:17-23.

Broadbent E, Ellis CJ, Thomas J, Gamble G, Petrie KJ: Can an illness perception intervention reduce illness anxiety in spouses of myocardial infarction patients? A randomized controlled trial. J Psychosom Res 2009b;67: 11-15.

Cameron LD, Leventhal HL: The Self-Regulation of Health and Illness Behaviour. London, Routledge, 2003.

Cohen L, Parker PA, Vence L, Savary C, Kentor D, et al: Presurgical stress management improves postoperative immune function in men with prostate cancer undergoing radical prostatectomy. Psychosom Med 2011;73: 218-225.

Constantino MJ, Arnkoff DB, Glass CR, Ametrano RM Smith JZ: Expectations. J Clin Psychol 2011;67:184192.

Enck P, Bingel U, Schedlowski M, Rief W: The placebo response in medicine: minimize, maximize or personalize? Nat Rev Drug Discov 2013;12:191-204.

Figueiras MJ, Weinman J: Do similar patient and spouse perceptions of myocardial infarction predict recovery? Psychol Health 2003;18:201-216.

Furze G, Dumville JC, Miles JN, Irvine K, Thompson DR, Lewin RJP: «Prehabilitation» prior to CABG surgery improves physical functioning and depression. Int $\mathrm{J}$ Cardiol 2009;132:51-58.

Go AS, Mozaffarian D, Roger VL, Benjamin EJ, Berry JD, et al: Heart disease and stroke statistics - 2014 update: a report from the American Heart Association. Circulation 2014;129:1-268.

Guo P: Preoperative education interventions to reduce anxiety and improve recovery among cardiac surgery patients: a review of randomised controlled trials. J Clin Nurs 2015;24:34-46.
Hauptman PJ, Chibnall JT, Guild C, Armbrecht ES: Patient perceptions, physician communication, and the implantable cardioverter-defibrillator. JAMA Intern Med 2013;173:571-577.

Hawkes AL, Nowak M, Bidstrup B, Speare R: Outcomes of coronary artery bypass graft surgery. Vasc Health Risk Manag 2006;2:477-484.

Hirani SP, Patterson DLH, Newman SP: What do coronary artery disease patients think about their treatments? An assessment of patients' treatment representations. J Health Psychol 2008;13:311-322.

Jonas WB, Crawford C, Colloca L, Kaptchuk TJ, Moseley $\mathrm{B}$, et al: To what extent are surgery and invasive procedures effective beyond a placebo response? A systematic review with meta-analysis of randomised, sham controlled trials. BMJ Open 2015;5:e09655.

Juergens MC, Seekatz B, Moosdorf RG, Petrie KJ, Rief W: Illness beliefs before cardiac surgery predict disability, quality of life, and depression 3 months later. J Psychosom Res 2010;68:553-560.

Kidd T, Poole L, Leigh E, Ronaldson A, Jahangiri M, Steptoe A: Health-related personal control predicts depression symptoms and quality of life but not health behaviour following coronary artery bypass graft surgery. J Behav Med 2016;39:120-127.

Kirsch I: Der Placeboeffekt in der antidepressiven Behand lung. Verhaltenstherapie 2016;26:55-61.

Krohne HW, El-Giamal M: Psychologische Operationsvor bereitung, Stressbewältigung und perioperativer Status. Z Gesundheitspsychol 2008;16:183-195.

Ladwig I, Rief W, Nestoriuc Y: Welche Risiken und Nebenwirkungen hat Psychotherapie? - Entwicklung des Inventars zur Erfassung Negativer Effekte von Psychotherapie (INEP). Verhaltenstherapie 2014;24:252-263.

Laferton JAC, Kube T, Salzmann S, Auer CJ, SheddenMora MC: Patients' expectations regarding medical treatment: a critical review of concepts and their assessment. Front Psychol 2017;8:233.

Laferton JAC, Shedden Mora M, Auer CJ, Moosdorf R, Rief W: Enhancing the efficacy of heart surgery by optimizing patients' preoperative expectations: study protocol of a randomized controlled trial. Am Heart J 2013;165:1-7.

Leventhal H, Meyer D, Nerenz DR: The common sense representation of illness danger; in Rachman S (ed): Contributions to Medical Psychology. New York, Pergamon Press, 1980, pp 7-30.

Lindsay GM, Smith LN, Hanlon P, Wheatley DJ: Coronary artery disease patients' perception of their health and expectations of benefit following coronary artery bypass grafting. J Adv Nurs 2000;32:1412-1421.
Murray CJL, Lopez AD: Measuring the global burden of disease. N Engl J Med 2013;369:448-457.

Pedersen SS, Knudsen C, Dilling K, Sandgaard NCF, Johansen JB: Living with an implantable cardioverter defibrillator: patients' preferences and needs for information provision and care options. Europace 2017;19: 983-990.

Rief W, Glombiewski JA: Erwartungsfokussierte Psychotherapeutische Interventionen (EFPI). Verhaltenstherapie 2016;26:47-54

Rief W, Shedden-Mora M, Laferton JA, Auer C, Petrie KJ et al: Preoperative optimization of patient expectations improves long-term outcome in heart surgery patients: results of the randomized controlled PSY-HEART trial. BMC Med 2017; 15:4.

Salzmann S, Euteneuer F, Laferton JAC, Auer CJ, SheddenMora MC, et al: Effects of preoperative psychological interventions on catecholamine and cortisol levels after surgery in coronary artery bypass graft patients: the randomized controlled PSY-HEART trial. Psychosom Med 2017;79:806-814.

Schedlowski M, Enck P, Rief W, Bingel U: Neuro-biobehavioral mechanisms of placebo and nocebo responses: implications for clinical trials and clinical practice. Pharmacol Rev 2015;67:697-730.

Scheier MF, Matthews KA, Owens JF, Schulz R, Bridges MW, et al: Optimism and rehospitalization after coronary artery bypass graft surgery. Arch Intern Med 1999;159:829-835.

Shedden-Mora M, Nestoriuc Y, Rief W: Lessons learned from placebo groups in antidepressant trials. Philos Trans R Soc Lond B Biol Sci 2011;366:1879-1888.

Tait RC, Chibnall JT, Krause S: The Pain Disability Index: psychometric properties. Pain 1990;40:171-182.

The Society of Thoracic Surgeons: What to Expect after Heart Surgery. https://sts.org/sites/default/files/whattoexpect.pdf, 2009 (letzter Abruf 28. Mai 2018).

Wampold BE: How important are the common factors in psychotherapy? An update. World Psychiatry 2015;14: 270-277.

Wampold BE, Minami T, Tierney SC, Baskin TW, Bhati KS: The placebo is powerful: estimating placebo effects in medicine and psychotherapy from randomized clinical trials. J Clin Psychol 2005;61:835-854.

Weinman J, Petrie KJ, Sharpe N, Walker S: Causal attributions in patients and spouses following first-time myocardial infarction and subsequent lifestyle changes. $\mathrm{Br} J$ Health Psychol 2000;5:263-273. 